\title{
A Load-Lock-Compatible Nanomanipulation System for Scanning Electron Microscope
}

\author{
Yan Liang Zhang*, Member, IEEE, Yong Zhang*, Student Member, IEEE, Changhai Ru*, Member, IEEE, \\ Brandon K. Chen, Student Member, IEEE, and Yu Sun, Senior Member, IEEE
}

\begin{abstract}
This paper presents a nanomanipulation system for operation inside scanning electron microscopes (SEM). The system is compact, making it capable of being mounted onto and demounted from an SEM through the specimen-exchange chamber (load-lock) without breaking the high vacuum of the SEM. This advance avoids frequent opening of the high-vacuum chamber, thus, incurs less contamination to the SEM, avoids lengthy pumping, and significantly eases the exchange of end effectors (e.g., nanoprobes and nanogrippers). The system consists of two independent 3-DOF Cartesian nanomanipulators driven by piezomotors and piezoactuators. High-resolution optical encoders are integrated into the nanomanipulators to provide position feedback for closed-loop control. The system is characterized, yielding the encoders' resolution of $2 \mathrm{~nm}$ and the piezoactuators' resolution of $0.7 \mathrm{~nm}$. A look-then-move control system and a contact-detection algorithm are implemented for horizontal and vertical nanopositioning.
\end{abstract}

Index Terms-Contact detection, nanomanipulation system, scanning electron microscope (SEM), system characterization, visual servoing.

\section{INTRODUCTION}

T HE CAPABILITY of real-time imaging with nanometer resolutions makes scanning electron microscopes (SEM) an appealing imaging platform for robotic manipulation of nanometer-sized objects. Nanomanipulation in an SEM has been used to manipulate carbon nanotubes [1]-[4], InGaAs/GaAs nanosprings [5], [6], and silicon nanowires [7]-[9] for characterizing their mechanical and/or electrical properties. Nanoassembly in an SEM was also demonstrated for the construction of three-dimensional (3-D) photonic crystals [10].

A number of nanomanipulation systems have been developed by companies, such as Kleindiek, Zyvex, SmarAct, and Attocube, as well as by academic laboratories (e.g., [1], [11] [13]). Piezomotors and piezoactuators are used due to their high

Manuscript received March 27, 2011; revised June 15, 2011; accepted August 13, 2011. Date of publication September 29, 2011; date of current version September 12, 2012. Recommended by Technical Editor Y. Li. This work was supported by Hitachi High-Technologies Canada Inc., by the Natural Sciences and Engineering Research Council of Canada, by the Ontario Centers of Excellence, and by the Canada Research Chairs Program.

*These authors contributed equally.

Y. L. Zhang, Y. Zhang, B. K. Chen, and Y. Sun are with the Advanced Micro and Nanosystems Laboratory, University of Toronto, Toronto, ON M5S 3G8, Canada (e-mail: yanliang.zhang@utoronto.ca; yzhang@mie.utoronto.ca; brandon.chen@utoronto.ca; sun@mie.utoronto.ca).

$\mathrm{C}$. Ru is with the Robotics and Microsystems Center, Soochow University, Suzhou, 215021, China (e-mail: changhai.ru@utoronto.ca).

Color versions of one or more of the figures in this paper are available online at http://ieeexplore.iee.org.

Digital Object Identifier 10.1109/TMECH.2011.2166162 positioning resolution, fast response, high force generation, and no magnetic-field generation (thus, no interference with electromagnetic imaging).

When a piezoelectric element is operated in the stick-slip mode for achieving a large motion range (e.g., millimeters), it is often called a piezo motor. The same piezoelectric element can also be operated in the fine mode (called a piezo actuator) to produce fine motion of nanometers with a travel range limited to a few micrometers. Using a single piezo element for both coarse and fine positioning is advantageous when a compact system size is desired. On the other hand, when employing two piezoelectric elements, one functioning as a piezo motor and the other independently as a piezo actuator, a nanomanipulation system is capable of extending the travel range of fine positioning from a few micrometers to tens of micrometers.

For installing a nanomanipulation system into an SEM, nanomanipulators are mounted onto, in most cases, the SEM stage [13]-[15], or less commonly, the chamber wall [16] or the ceiling [17] of the SEM. SEM imaging and nanomanipulation occur in the high-vacuum chamber. The installation of a nanomanipulation system or exchanging end effectors (e.g., nanoprobes and nanogrippers) requires the opening of the highvacuum chamber. Breaking the high vacuum and exposing the chamber to the ambient environment contaminate the chamber and incur a lengthy pump-down process (e.g., $30 \mathrm{~min}$ to $2 \mathrm{~h}$ ) after closing the chamber.

Besides the high-vacuum chamber, an SEM has a specimenexchange chamber (typically much smaller than the highvacuum chamber) through which a specimen is transferred into and out of the high-vacuum chamber. The specimen transfer process does not significantly alter the high vacuum in the SEM main chamber; thus, no lengthy pumping is required, and less contamination is incurred in comparison to the opening of the high-vacuum chamber. Therefore, it is desirable to develop a compact nanomanipulation system that can be transferred through the specimen-exchange chamber without breaking the high vacuum. This advance would make nanomanipulation systems more SEM-compatible and improve productivity especially for nanomanipulation tasks that require frequent exchange of end effectors.

SEM nanomanipulation has been largely performed manually by an operator using a joystick and/or a keypad, while closely monitoring SEM images, which is time consuming and skill dependent, and results in low productivity and frequent end-effector breakage. Using the SEM as a vision sensor, advances were recently made [12], [18]-[21] to facilitate automated nanopositioning. However, piezomotor or piezoactuator 


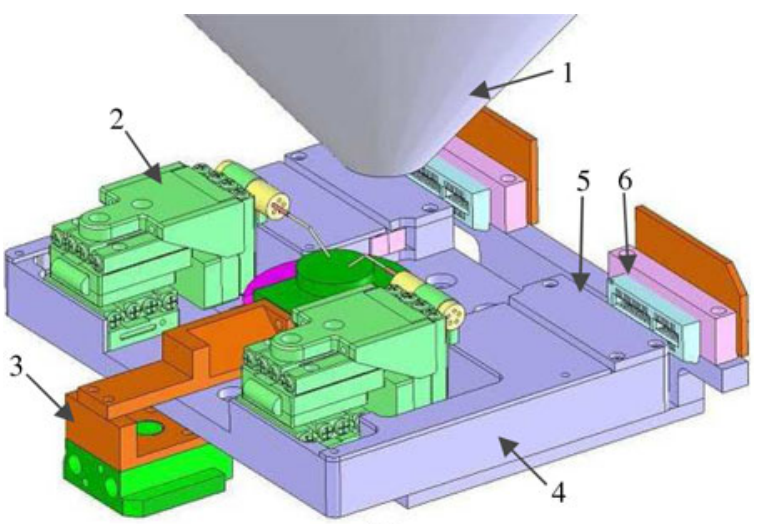

(a)

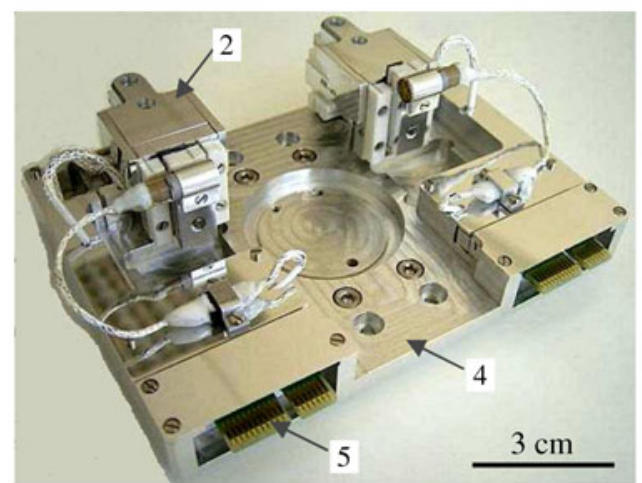

(b)

Fig. 1. Nanomanipulation system that is compact in size, capable of being transferred through the specimen-exchange chamber of a standard SEM: 1-SEM pole piece; 2-nanomanipulator; 3-specimen holder; 4nanomanipulator carrier; 5-male electrical connectors; and 6-female electrical connector.

has inherent hysteresis demanding a high-bandwidth sensor for hysteresis modeling in feedforward control [22] or closed-loop control [23]-[26], but SEM imaging suffers from low frame rates, image drift, and noise, and image-quality variations due to environmental factors and electrical charging of specimens, necessitating the use of additional position feedback, such as high-resolution optical encoders.

This paper reports a nanomanipulation system (see Fig. 1) that is small in size, capable of being transferred through the specimen-exchange chamber of a standard SEM. This feature circumvents the opening of the high-vacuum chamber of the SEM, which is necessary for existing nanomanipulation systems. The system also integrates high-resolution optical encoders to provide position feedback along multiple axes with nanometer resolutions for closed-loop positioning. The system design, system characterization details, and system performance are presented.

\section{SYSTEM DESIGN}

As shown in Fig. 1, the nanomanipulation system has a nanomanipulator carrier (4), on which there are two nanomanipulators (2) and two corresponding male electrical connectors (5). The female electrical connectors (6) are installed onto the SEM main chamber stage permanently, and mate with connectors (5)

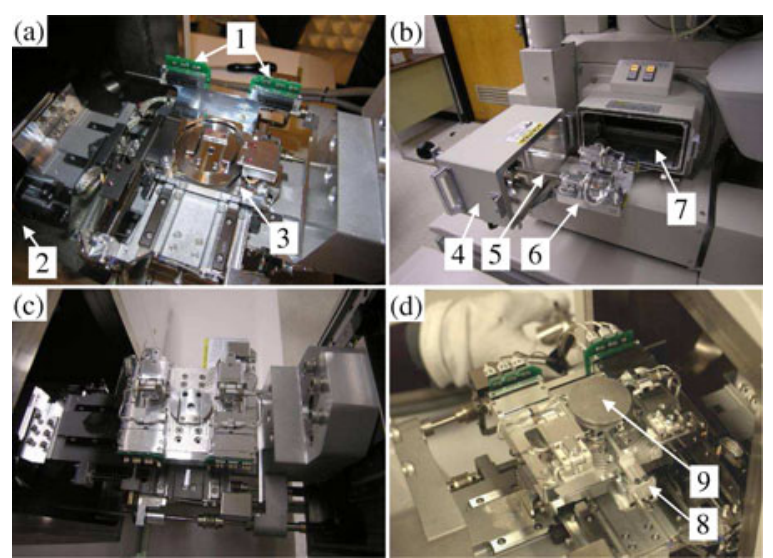

Fig. 2. Installation procedure of the nanomanipulation system. (a) Female electrical connectors (1) are installed to the specimen stage (3) inside the SEM main chamber (2). (b) The nanomanipulator carrier (6) is installed to the specimen-exchange rod (5) after the specimen-exchange chamber (7) is aired and its door (4) is opened. (c) The nanomanipulator carrier is transferred to the main chamber for mechanical mounting to the specimen stage and electrical coupling to the previously installed female electrical connectors. (d) The specimen holder (8) with a specimen stub (9) is transferred to the manipulator carrier through the specimen-exchange chamber.

when carrier (4) is transferred through the specimen-exchange chamber like a regular specimen. Electrical wires from connectors (6) are connected to the nanomanipulator driver/controller outside the SEM through an SEM feedthrough port. Carrier (4) has a T-shaped structure at the bottom for its mounting to the T-groove of the specimen stage. After the transfer of carrier (4), the specimen holder (3) is transferred to the carrier through the specimen-exchange chamber.

The installation of electrical connectors requires one-time opening of the high-vacuum chamber, while mounting and demounting of nanomanipulators do not break the high vacuum nor does the exchange of end effectors affect the high vacuum. In addition, in this design architecture, transferring a specimen into and out of the SEM is decoupled from the nanomanipulation system. Thus, during specimen exchanging, the nanomanipulation system stays inside the SEM without being disturbed. Fig. 2 shows the installation procedure of the nanomanipulation system to a standard SEM (Hitachi SU6600). For demounting the specimen holder or the nanomanipulator carrier, the specimenexchange rod is used again to transfer them out through the specimen-exchange chamber.

The system contains two nanomanipulators, both assembled from three nanopositioners (SmarAct $\mathrm{GmbH}$; http://www.smaract.de/) integrated with optical encoders (reported resolution: $1 \mathrm{~nm}$, Numerik Jena $\mathrm{GmbH}$ ). The size of each nanomanipulator is $36 \times 21 \times 22 \mathrm{~mm}^{3}$. The nanopositioners, as estimated by the manufacturer, when operating in the coarse positioning mode (i.e., as a piezomotor), have a travel range of $10 \mathrm{~mm}$ and a resolution of $\sim 50 \mathrm{~nm}$, and when operating in the fine positioning mode (i.e., as a piezoactuator), have a travel range of $1 \mu \mathrm{m}$ and a resolution of $\sim 1 \mathrm{~nm}$. The load carrying capability of the nanopositioner is approximately $3 \mathrm{~N}$.

The system can be redesigned to contain more than two nanomanipulators. Additionally, it is also feasible to integrate 


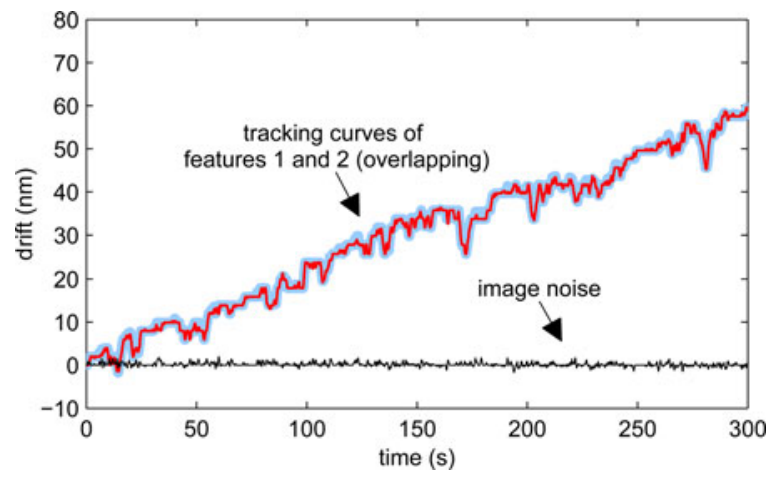

Fig. 3. Quantification of SEM image drift and noise. Drift curves (top) were collected by visually tracking two stationary features on the specimen. Noise curve (bottom) results from the substraction of the two drift curves.

nanomanipulators with both translational and rotational degrees of freedom into the system for more dexterous nanomanipulation. The present nanomanipulation system producing only translational motions was systematically characterized to quantify its performance inside the SEM.

\section{SYSTEM CHARACTERIZATION}

\section{A. SEM Imaging}

SEM imaging is used as a benchmark to characterize the performance of the actuators and encoders of the nanomanipulation system. The SEM is known to have issues, such as image drift and noise, making stationary features on a specimen appear moving in the image frame. Thus, the characterization of drift and noise in SEM imaging was conducted.

SEM image noise refers to random variations of pixel values, arising from a few sources (e.g., limited number of excited secondary electrons from specimens). Image drift refers to the movement of the entire image due to electron beam drift, charge drift on a specimen, and/or electromagnetic interferences from the environment. In order to quantify image drift and noise, two features on a stationary specimen were visually tracked simultaneously over 5 min using a subpixel visual-tracking algorithm [27]. A high imaging magnification of $100000 \times$ was used along with other optimal imaging conditions (e.g., a short working distance of $5 \mathrm{~mm}$, a high acceleration voltage of $30 \mathrm{kV}$, and the use of a magnetic field cancellation device). The pixel size at $100000 \times$ magnification is $2 \mathrm{~nm} \times 2 \mathrm{~nm}$. These imaging conditions were also used in the subsequent characterization of the nanomanipulation system.

Each of the two tracking curves in Fig. 3 represents the position change of a feature relative to its original position at 0 $\mathrm{s}$ in the image. Those two tracked features were $1 \mu \mathrm{m}$ apart. The two curves essentially overlap, indicating that the image drift dominates noise. The relative position change between the two features (subtraction between the two tracking curves) represents image noise, shown as the horizontal curve in Fig. 3 (mean: $0.05 \mathrm{~nm}$; standard deviation: $0.33 \mathrm{~nm}$ ).

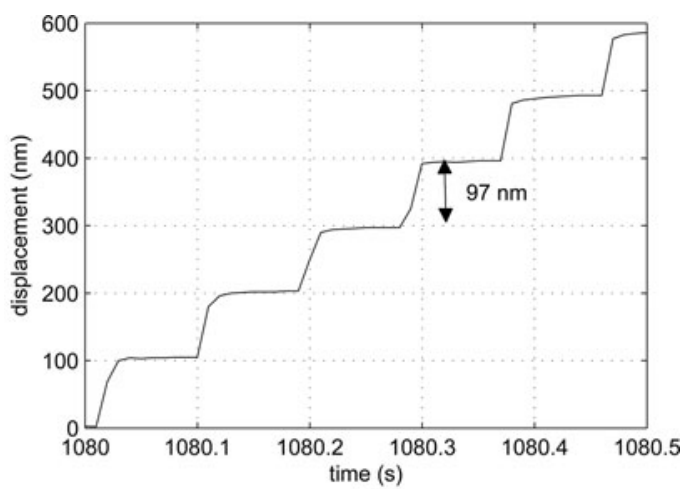

(a)

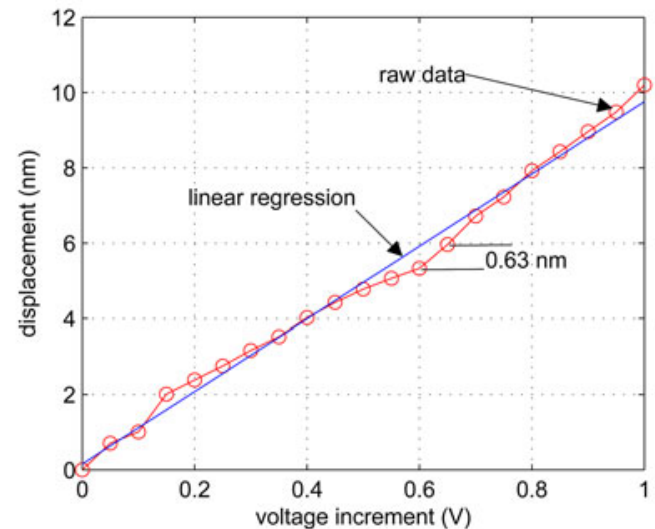

(b)

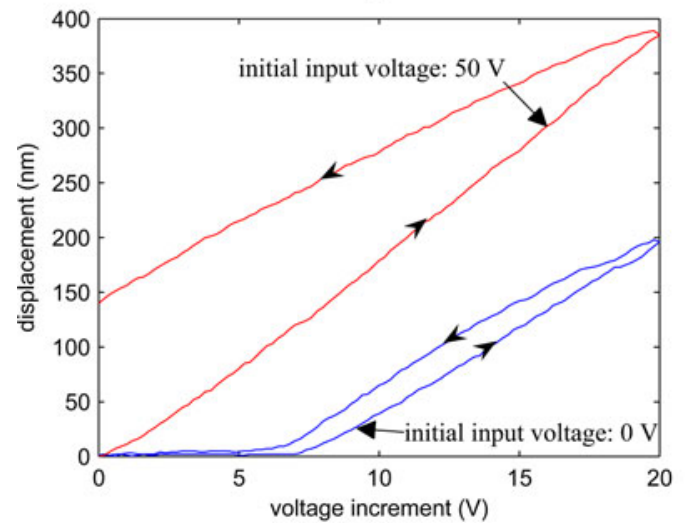

(c)

Fig. 4. Actuator characterization results. (a) Step size of the stick-slip movement was experimentally determined. The minimum step size that the system is capable of producing with a high repeatability is determined to be $97.2 \mathrm{~nm}$ with a standard deviation of $1.1 \mathrm{~nm}$. (b) Resolution of the actuator is characterized to be better than $0.70 \mathrm{~nm}$. (c) Hysteretic behaviors of the piezoactuator when the initial input voltages are 0 and $50 \mathrm{~V}$, respectively.

\section{B. Actuator Characterization}

The step size of the piezomotor (stick-slip mode) in coarse movement depends on the amplitude and frequency of the driving sawtooth signal. The minimum step size is defined as the minimum repeatable movement of the actuator in the coarse mode. As shown in Fig. 4(a), the minimum step size of the nanomanipulators is $97.2 \mathrm{~nm}$ with a standard deviation of 1.1 $\mathrm{nm}$ when the amplitude and frequency of the driving sawtooth signal are set to be $48.84 \mathrm{~V}$ and $12000 \mathrm{~Hz}$, respectively. 
The input voltage of the piezoactuator ranges from 0 to $100 \mathrm{~V}$ using a 12-bit digital-to-analog converter (DAC), corresponding to $10 \mathrm{~nm} / \mathrm{V}$. The piezoactuator often starts at its center position, e.g., 50-V actuation voltage, in a coarse-fine positioning system for the purpose of producing forward/backward movement. The piezoactuator was driven by an input voltage increasing from 50 to $51 \mathrm{~V}$ with an interval of $0.024 \mathrm{~V}$ (i.e., $100 / 2^{12} \mathrm{~V}$ ). The same imaging conditions and subpixel visual-tracking algorithm as used in the SEM characterization were applied. As shown in Fig. 4(b), the subpixel visual-tracking algorithm detects the movement of the piezoactuator after each two-voltage increments, since one-voltage increment results in the calculated movement of $0.24 \mathrm{~nm}$, smaller than the SEM imaging noise level (i.e., $0.33 \mathrm{~nm}$ ). The mean and standard deviation of the position increments are 0.51 and $0.19 \mathrm{~nm}$, respectively, indicating that piezoactuator has a resolution better than $0.70 \mathrm{~nm}$.

As the actuation voltage increases by $20 \mathrm{~V}$ and returns to the initial voltage, the piezoactuator exhibits significant hysteretic behavior [see Fig. 4(c)], translating into poor linearity between the displacement and the actuation voltage. For portions of the curve, where a voltage increment corresponds to a travel distance larger than the image noise level, one-voltage increment can also result in a movement detectable by the tracking algorithm. Fig. 4(c) also indicates that hysteresis is highly dependent on the starting voltage, necessitating closed-loop control [23], [25], [26] or a suitable mathematical model of the hysteretic behavior [28]-[36] to compensate for the nonlinearity.

\section{Encoder Characterization}

Drift, accuracy, and resolution of the optical encoders were characterized inside the SEM. The encoder reading drifts because the light source of the encoder generates heat and causes the optical scale of encoder to expand. This issue is worsened by poor heat transfer in the high-vacuum environment of the SEM. Fig. 5(a) shows encoder reading sampled at $100 \mathrm{~Hz}$ for $10 \mathrm{~min}$, while the nanomanipulators were kept unactuated. It is not clear why the encoders drift in one direction for the first few seconds when they are turned $\mathrm{ON}$ and, subsequently, drift in the opposite direction. After the direction reverses, all encoders drift at a near constant rate of $1.2 \mathrm{~nm} / \mathrm{s}$. This result is useful for encoder-drift compensation when required. It also guides one to limit the duration between two critical encoder readings to below $1 \mathrm{~s}$, in order to avoid/mitigate the effect of encoder drift.

Encoder accuracy was quantified using SEM imaging. The nanomanipulators were moved in the $X Y$ plane in the open loop, with the travel distance measured from both the encoders and SEM imaging. The absolute accuracy of the encoders depends on travel distances. For example, when the nanomanipulator traveled by $1292 \mathrm{~nm}$ (measured by SEM imaging), the corresponding encoder reading was $1280 \mathrm{~nm}$. The encoder error changes proportionally with traveled distances. The results show that the average accuracy of the encoders is $98 \%$ of SEM measurements.

Subtracting the drift (e.g., a ramp signal of $1.2 \mathrm{~nm} / \mathrm{s}$ ) from the encoder reading in Fig. 5(a) yields the noise component of the reading, as shown in Fig. 5(b). The noise has a $p-p$ value

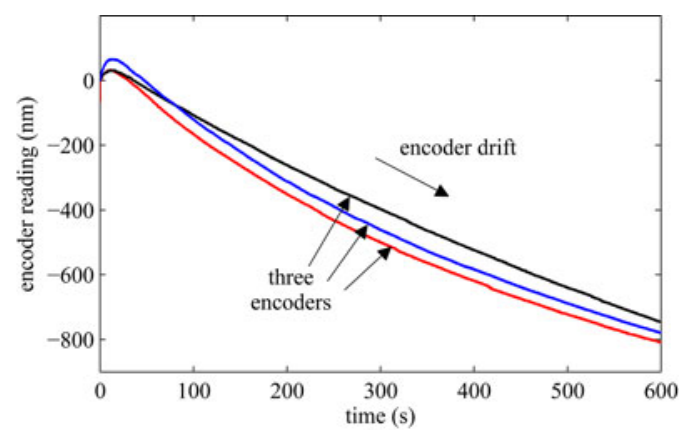

(a)

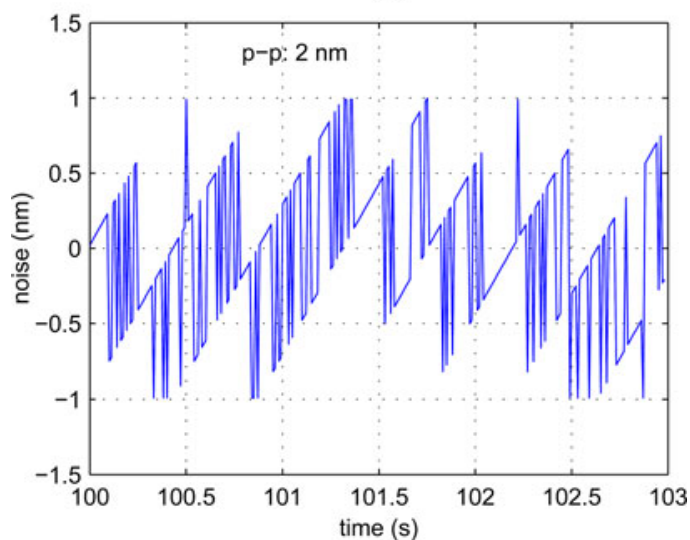

(b)

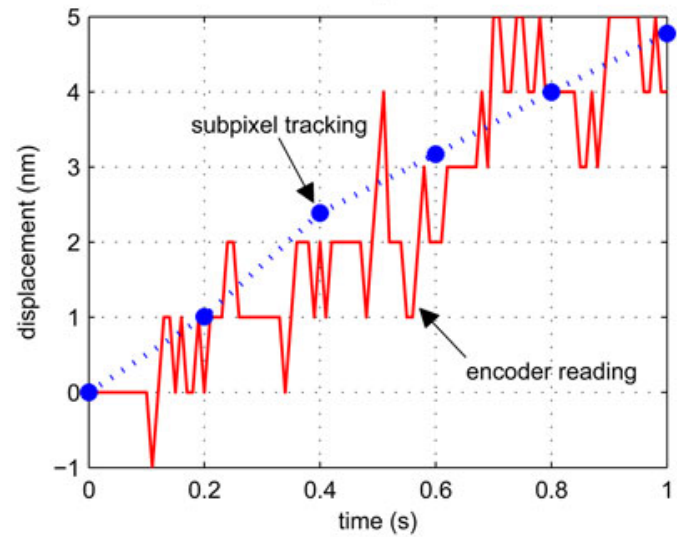

(c)

Fig. 5. Encoder characterization results. (a) Drift quantification. (b) Noise quantification. (c) Resolution quantification.

of $2 \mathrm{~nm}$, limited by the resolution of the encoder. To quantify the minimum motion that an encoder (i.e., encoder resolution) is able to detect, a piezoactuator (fine mode) was actuated to travel by approximately $5 \mathrm{~nm}$ during $1 \mathrm{~s}$ with an interval of 1 $\mathrm{nm}$. The subpixel visual-tracking result from SEM imaging is deemed to be the actual displacement, and the encoder reading is the measured output. As shown in Fig. 5(c) and Table I, the difference between the actual position and the encoder reading is less than $2 \mathrm{~nm}$, demonstrating that the encoder has a $2-\mathrm{nm}$ resolution.

It should be noted that when an actuator was actuated to travel a small distance close to the resolution of its encoder, the encoder resolution is the major factor determining the accuracy the encoder reading. When the traveled distance is much larger 
TABLE I

RESOLUTION OF THE OPTICAL ENCODERS

\begin{tabular}{c|c|c|c|c|c|c}
\hline subpixel $(\mathrm{nm})$ & 0 & 1.01 & 2.39 & 3.17 & 4.0 & 4.78 \\
\hline encoder (averaged) $(\mathrm{nm})$ & 0 & 0 & 2 & 2 & 4 & 4 \\
\hline (subpixel - encoder) $(\mathrm{nm})$ & 0 & 1.01 & 0.39 & 1.17 & 0 & 0.78 \\
\hline
\end{tabular}

than the encoder resolution, the effect of the encoder accuracy alone dominates the effect of the encoder resolution.

\section{Control}

The integration of encoders enables the implementation of the look-then-move control in the $X Y$ directions. Considering the large travel range of the piezomotor in the coarse mode and the small travel range of the piezoactuator in the fine mode, two classical discrete proportional-integral differential (PID) controllers were implemented to control the coarse-fine system. A switch function was used to determine the selection of the two PID controllers.

However, the accuracy of the optical encoders (e.g., 98\% over the travel range) degrades the accuracy of position control in the image frame, demanding an image-based visual servo integrated with the look-then-move control. The combined use of the lookthen-move and image-based visual servo improves the accuracy while maintaining a reasonable speed.

Denoting $f_{d} \in \Re^{2 \times 1}, f_{i} \in \Re^{2 \times 1}$, and $f_{c} \in \Re^{2 \times 1}$ as the desired, initial, and current positions of the end effector in the image frame, and $X_{i} \in \Re^{2 \times 1}$ as the initial position along the $X Y$ directions in the actuator coordinate system, the desired position $X_{d} \in \Re^{2 \times 1}$ of the end effector in the actuator coordinate system is

$$
X_{d}=J\left(f_{d}-f_{i}\right)+X_{i}
$$

where $J \in \Re^{+}$is the ratio between the displacements in the image frame and the actuator coordinate system by assuming that $X$ and $Y$ axes of the actuator coordinate system coincide with column and row of the image frame, respectively. The assumption is feasible because the electron beam and the SEM sample stage can be rotated to algin the $X$ and $Y$ axes with the column and row of the image frame.

1) Coarse-Fine Switch of the Look-Then-Move Control: The control input (e.g., $U_{x}$ along the $X$ direction) is switched from the coarse mode $U_{c}$ to the fine mode $U_{f}$ by comparing the error $E_{x}$ with the travel range $\Theta$ of the piezoactuator as follows:

$$
U_{x}=\left\{\begin{array}{l}
U_{c}, E_{x}>\Theta \\
U_{f}, E_{x} \leq \Theta
\end{array}\right.
$$

where $U_{c}$ and $U_{f}$ are the control outputs from classical discrete PID controllers

$$
\begin{aligned}
& U_{c}=K_{p c} E_{x}(n)+K_{i c} \sum_{k=0}^{n} E_{x}(k)+K_{d c} \Delta(n) \\
& U_{f}=K_{p f} E_{x}(m)+K_{i f} \sum_{k=0}^{m} E_{x}(k)+K_{d f} \Delta(m)
\end{aligned}
$$

where $n(m)$ is the discrete step at time $t, K_{p c}, K_{i c}, K_{d c}$ and $K_{p f}, K_{i f}, K_{d f}$ are the PID parameters for the coarse and fine control, respectively. $E_{x}(n)\left(E_{x}(m)\right)$ is the error between the current position and the desired position, and $\Delta(n)(\Delta(m))$ is the difference between the current position and the previous position at time $n-1(m-1)$.

2) Coarse-Fine Switch of the Image-Based Visual Servo: The form of the controller of coarse-fine image-based visual servo is similar as (3) and (4), except that the error is determined in the image frame. The switch of the coarse-fine control input $u_{x}$ is as follows:

$$
u_{x}= \begin{cases}u_{c}, & e_{x}>\Omega \\ u_{f}, & e_{x} \leq \Omega\end{cases}
$$

where $u_{c}$ and $u_{f}$ are the coarse and fine control inputs, and $\Omega$ (pixel) is often chosen as the ratio between the travel range of the piezoactuator and the pixel size of the SEM image.

3) Switch of the Look-Then-Move Control and Image-Based Visual Servo: The image-based visual servo will not be triggered until the look-then-move control reaches its steady state, e.g., $E_{x}=\tau \mathrm{nm}$ (the absolute accuracy of the optical encoder corresponding to a travel distance) by choosing the PID parameters in $U_{c}$ and $U_{f}$ carefully. For example, the control input $u$ in the $X$ direction is

$$
u=\left\{\begin{array}{cl}
U_{x}, & \left|E_{x}\right|>\tau \\
u_{x}, & E_{x} \leq \tau .
\end{array}\right.
$$

4) Positioning Along the $Z$ Direction: The $Z$ position of a nanomanipulator can also be obtained through SEM imaging, by tilting the SEM stage to achieve views from a different angle. The tilting angle can be determined from the rotary encoder of the SEM specimen stage. The stage tilting induces relative position change between the end effector and the substrate in the image frame. With the tilting angle and position change being known, the height of the end effector relative to the substrate surface can be calculated. However, this method depends on the availability of high-resolution rotary encoders and is time costly in implementation. Thus, an SEM vision-based contactdetection method is developed for $Z$-position determination.

Accurately detecting the contact between an end effector and the substrate can provide a nanomanipulation system with a reference along the $Z$ direction. We extended our vision-based contact-detection algorithm from optical microscopy [37], [38] to electron microscopy [20]. This approach does not require additional equipment, devices, or sensors, different from approaches reported previously [15], [18], [39]- [41]. Using the SEM visual feedback, the end effector (i.e., nanoprobe) is visually tracked in real time as it descends toward the target surface. Once contact between the end effector and target surface is established, further motion of the end effector in the $Z$ direction causes the end effector to slide on the substrate surface. This sliding motion is detected by comparing the pixel values difference (e.g., $x_{i}-x_{i-5}$ ) and a threshold $\Delta d$ (e.g., four pixels), as shown in Fig. 6.

Upon the detection of the contact (e.g., point B in Fig. 6), the end effector is retracted to the actual contact point (point $\mathrm{C}$ ) by ascending in the $Z$ direction by $\Delta Z$ and, then, raised by tens of nanometers $\left(\Delta Z_{a}\right)$ (to point $\mathrm{D}$ ). As summarized in Table II, the encoder accuracy is within $98 \%$ of travel distance. Since 


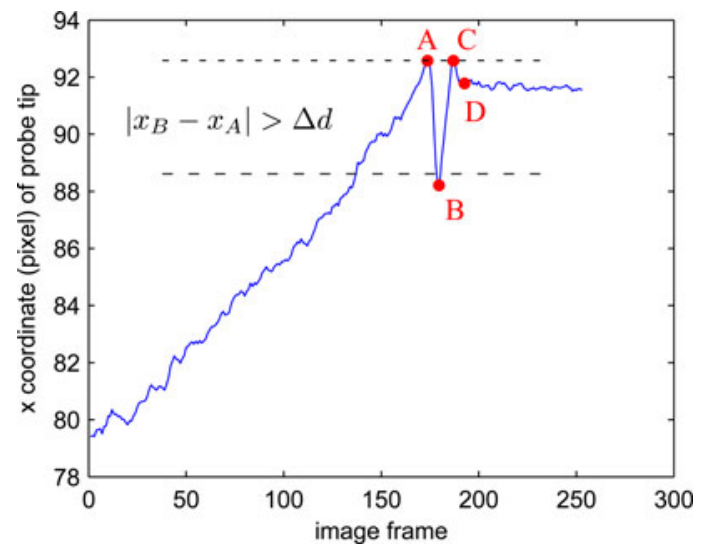

Fig. 6. End effector slides on the substrate after a contact is established. A contact is detected if the pixel values difference in current $x_{i}$ (e.g., B) and previous position $x_{i-5}$ (e.g., A) is greater than a threshold $\Delta d$ (e.g., four pixels).

TABLE II

SUMMARY OF SySTEM PERFORMANCE

\begin{tabular}{c|c|c}
\hline \multirow{2}{*}{ dimension } & complete system & $100 \times 80 \times 46 \mathrm{~mm}^{3}$ \\
\cline { 2 - 3 } encoder & manipulator & $36 \times 21 \times 22 \mathrm{~mm}^{3}$ \\
\cline { 2 - 3 } & resolution & $2 \mathrm{~nm}$ \\
\cline { 2 - 3 } & accuracy & $98 \%$ of travel distance \\
\hline \multirow{2}{*}{ piezo motor } & drift & $1.2 \mathrm{~nm} / \mathrm{s}$ \\
\cline { 2 - 3 } & range & $11 \mathrm{~mm}$ \\
\hline \multirow{2}{*}{ piezo actuator } & rep size & $1.2 \mu \mathrm{mm}$ \\
\cline { 2 - 3 } & resolution & $0.7 \mathrm{~nm}$ \\
\hline \multirow{3}{*}{ closed loop } & $\begin{array}{c}\text { accuracy } \\
\text { (look-then-move) }\end{array}$ & $98 \%$ of travel distance \\
\cline { 2 - 3 } & $\begin{array}{c}\text { accuracy } \\
\text { (visual servo) }\end{array}$ & $<1$ pixel \\
\hline
\end{tabular}

the motion range along the $\Delta Z$ is typically not beyond 100 $\mathrm{nm}$ after contact detection, the nanomanipulation system uses only encoder feedback and standard PID position control for positioning along the $Z$ direction after the first contact detection.

\section{RESULTS AND DISCUSSION}

Table II summarizes system specifications and characterized performance. The size of the overall system makes it easily fit through the SEM specimen-exchange chamber to enter the highvacuum chamber. The coarse positioning resolution of $97.2 \mathrm{~nm}$ is sufficient for the system to manipulate submicrometer objects. However, in order to manipulate nanometer-sized objects, the system must operate in the fine mode to produce a motion resolution of nanometers.

While the steady-state error of the closed-loop-controlled nanomanipulation system is determined by the resolution of the encoders, the positioning accuracy depends on the accuracy of the encoders. For example, when the system is controlled to travel $320 \mathrm{~nm}$ along the $X$ or $Y$ direction, the system reaches the target position with an error of $\pm 4 \mathrm{~nm}$, benchmarked by SEM imaging. The average encoder accuracy and actuator performance listed in Table II are also applicable to the $Z$ direction.

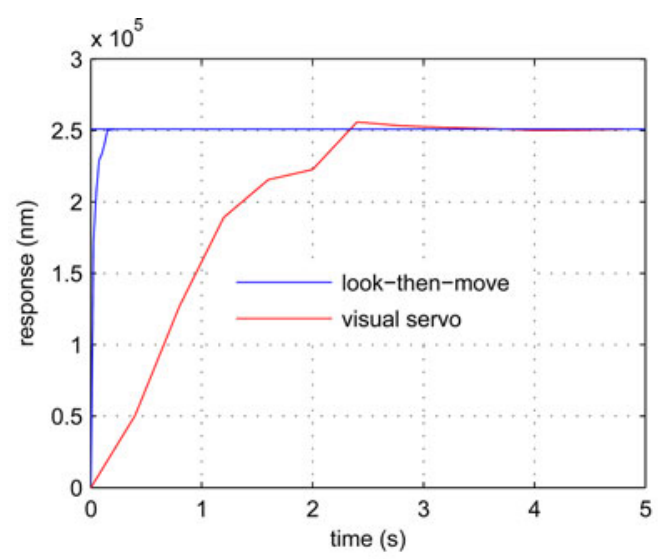

(a)

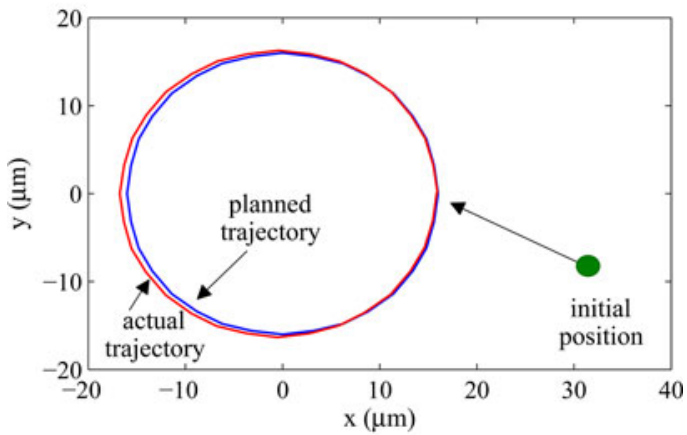

(b)

Fig. 7. Control system performance. (a) Step response of the system. Comparison of the look-then-move control and the image-based visual servo. (b) Tracking a circle using the look-then-move control.

Characterizing the system's positioning performance along the $Z$ direction was performed by accurately tilting the SEM stage and using SEM imaging from multiple oblique views.

The integrated encoders increase the system bandwidth. A comparison was made between the look-then-move control and the image-based visual servo. The encoder sampling frequency was set to $100 \mathrm{~Hz}$. SEM imaging at the fast scan mode is 30 $\mathrm{Hz}$ as the maximum. The control gains were tuned through trial and error. To travel a distance of $250 \mu \mathrm{m}$, the look-then-move control costs $0.25 \mathrm{~s}$ to reach the steady state, while visual servo takes 3 s, as shown in Fig. 7(a).

Fig. 7(b) shows the tracking of a circle (diameter $32 \mu \mathrm{m}$ ) with the look-then-move control approach. Tracking the full circle took the system 0.87 and $10.33 \mathrm{~s}$ using the look-then-move and image-based visual servo, respectively. Due to encoder inaccuracies ( $98 \%$ of the travel distance), the largest error generated by the system was approximately $640 \mathrm{~nm}$. It is also observed that the tracking errors were larger for target positions farther away from the original position, since the absolute encoder error is proportional to the travel distance.

In order to leverage the high speed of the look-then-move approach, while reducing its errors, a combined use of the lookthen-move and image-based visual servo was implemented. After the look-then-move control reaches the steady state, imagebased visual servo can be used to fine tune the final positions, making the tracking accuracy less than 1 pixel with a tradeoff of a longer tracking time ( $2.58 \mathrm{~s}$ versus $0.87 \mathrm{~s})$. 


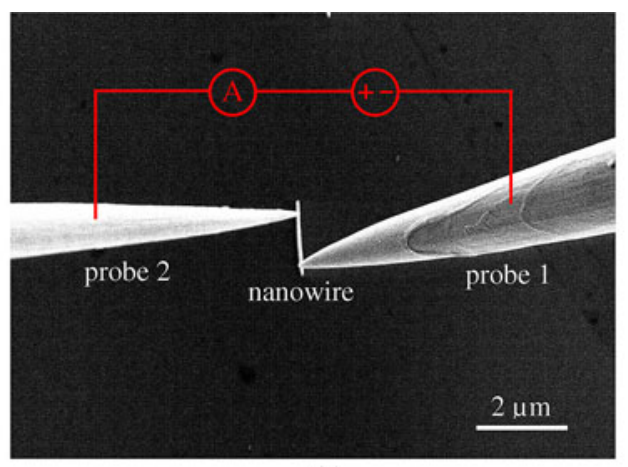

(a)

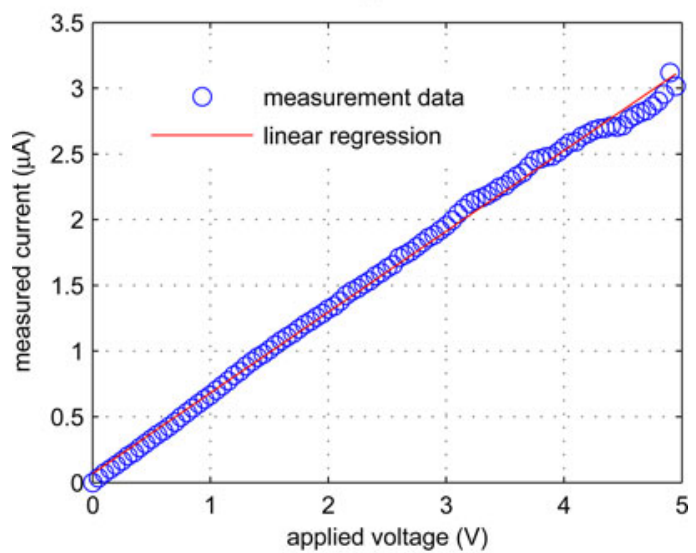

(b)

Fig. 8. Nanowire probing. (a) Two nanoprobes landing on a nanowire $(90$ $\mathrm{nm}$ in diameter) for electrical characterization. (b) $I-V$ characteristics of the probed nanowire.

A range of end effectors, such as nanoprobes, atomic force microscopy (AFM) cantilevers, and micro-nanogrippers can be mounted on the nanomanipulation system to perform tasks such as nanomaterial characterization and pick-place assembly. In this paper, we use nanowire probing (tin-oxide nanowires) as an example application to demonstrate the operation of the system.

Both nanomanipulators were mounted with a tungsten nanoprobe. An electrical connection between the nanoprobes and outside SEM was established through electrical connectors on the nanomanipulators and the SEM feedthrough port. The probes were chemically treated to remove the native oxide layer using $\mathrm{KOH}$ and $\mathrm{HF}$ solutions, having an end tip of approximately $150 \mathrm{~nm}$ in diameter.

The system is capable of detecting the contact position by monitoring the slope change in the image pattern. Contact detection typically costs $10 \mathrm{~s}$. The system positions the nanoprobes using the look-then-move control along the $X Y$ direction and position control along the $Z$ direction. Fig. 8(a) shows the probing of a 90-nm wide Sn nanowire. The exact accuracy of contact detection has not been fully characterized; however, the fact that the nanoprobes were able to repeatedly land on the $90 \mathrm{~nm}$ nanowire confirms that the contact-detection accuracy is better than $90 \mathrm{~nm}$. The average positioning time for probing a nanowire is $0.25 \mathrm{~s}$ along $X Y$ directions. A SourceMeter (2602, Keithley Instruments Inc., Solon, $\mathrm{OH}$ ) was used to supply voltages and measure currents. The resistivity of the nanowire was determined from the $I-V$ data shown in Fig. 8 (b) to be $1.10 \times$ $10^{-3} \Omega \cdot \mathrm{m}$.

\section{CONCLUSION}

This paper presented a compact, closed-loop-controlled nanomanipulation system. The system eases end-effector exchange without requiring the opening of the high-vacuum chamber of an SEM, eliminating lengthy pumping processes and incurs less contamination. The system was systematically characterized, demonstrating the suitability for nanopositioning. The integrated high-resolution encoders increase system bandwidth. When the encoder feedback and SEM visual feedback are combined, the control system is capable of achieving both a high speed and a high accuracy.

\section{REFERENCES}

[1] M.-F. Yu, O. Lourie, O. Lourie, K. Moloni, T. F. Kelly, and R. S. Ruoff, "Strength and breaking mechanism of multiwalled carbon nanotubes under tensile load," Science, vol. 287, pp. 637-640, 2000.

[2] T. Fukuda, F. Arai, and L. Dong, "Assembly of nanodevices with carbon nanotubes through nanorobotic manipulations," Proc. IEEE, vol. 91, no. 11, pp. 1803-1818, Nov. 2003.

[3] H. D. Espinosa, Y. Zhu, and N. Moldovan, "Design and operation of a MEMS-based material testing system for nanomechanical characterization," J. Microelectromech. Syst., vol. 16, pp. 1219-1231, 2007.

[4] T. Fukuda, M. Nakajima, P. Liu, and H. ElShimy, "Nanofabrication, nanoinstrumentation and nanoassembly by nanorobotic manipulation," Int. J. Robot. Res., vol. 28, pp. 537-547, 2009.

[5] D. J. Bell, L. Dong, B. J. Nelson, M. Golling, L. Zhang, and D. Grützmacher, "Fabrication and characterization of three-dimensional InGaAs/GaAs nanosprings," Nano Lett., vol. 6, pp. 725-729, 2006.

[6] G. Hwang, H. Hashimoto, D. J. Bell, L. Dong, B. J. Nelson, and S. Schön, "Piezoresistive InGaAs/GaAs nanosprings with metal connectors," Nano Lett., vol. 9, pp. 554-561, 2009.

[7] Y. Zhu, F. Xu, Q. Qin, W. Y. Fung, and W. Lu, "Mechanical properties of vapor-liquid-solid synthesized silicon nanowires," Nano Lett., vol. 9, pp. 3934-3939, 2009.

[8] A. Lugstein, M. Steinmair, A. Steiger, H. Kosina, and E. Bertagnolli, "Anomalous piezoresistance effect in ultrastrained silicon nanowires," Nano Lett., vol. 10, no. 8, pp. 3204-3208, 2010.

[9] Y. Zhang, X. Liu, C. Ru, Y. L. Zhang, L. Dong, and Y. Sun, "Piezoresistivity characterization of synthetic silicon nanowires using a MEMS device," J. Microelectromech. Syst., vol. 20, no. 4, pp. 959-967, Aug. 2011.

[10] K. Aoki, H. T. Miyazaki, H. Hirayama, K. Inoshita, T. Baba, K. Sakoda, N. Shinya, and Y. Aoyagi, "Microassembly of semiconductor threedimensional photonic crystals," Nat. Mater., vol. 2, pp. 117-121, 2003.

[11] L. Dong, F. Arai, and T. Fukuda, "Destructive constructions of nanostructures with carbon nanotubes through nanorobotic manipulation," IEEE/ASME Trans. Mechatronics, vol. 9, no. 2, pp. 350-357, Jun. 2004.

[12] S. Fatikow, T. Wich, H. Hülsen, T. Sievers, and M. Jähnisch, "Microrobot system for automatic nanohandling inside a scanning electron microscope," IEEE/ASME Trans. Mechatronics, vol. 12, no. 3, pp. 244-252, Jun. 2007.

[13] E. C. Heeres, A. J. Katan, M. H. van Es, A. F. Beker, M. Hesselberth, D. J. van der Zalm, and T. H. Oosterkamp, "A compact multipurpose nanomanipulator for use inside a scanning electron microscope," Rev Sci. Instrum., vol. 81, 023704, 2010.

[14] L. X. Dong, F. Arai, and T. Fukuda, "Electron-beam-induced deposition with carbon nanotube emitters," Appl.Phys. Lett., vol. 81, pp. 1919-1921, 2002.

[15] B. K. Chen, Y. Zhang, D. D. Perovic, and Y. Sun, "MEMS microgripper with thin gripping tips," J. Micromech. Microeng., vol. 21, no. 10, 105004, 2011.

[16] D. Zhang, J.-M. Breguet, R. Clavel, V. Sivakov, S. Christiansen, and J. Michler, "In situ electron microscopy mechanical testing of silicon nanowires using electrostatically actuated tensile stages," J. Microelectromech. Syst., vol. 19, pp. 663-674, 2010.

[17] M. Noyong, K. Blech, A. Rosenberger, V. Klocke, and U. Simon, "In situ nanomanipulation system for electrical measurements in SEM," Meas. Sci. Technol., vol. 18, pp. N84-N89, 2007.

[18] V. Eichhorn, S. Fatikow, T. Wich, C. Dahmen, T. Sievers, K. N. Andersen, K. Carlson, and P. Bøggild, "Depth-detection methods for microgripper based CNT manipulation in a scanning electron microscope," J. MicroNano Mech., vol. 4, pp. 27-36, 2008. 
[19] B. E. Kratochvil, L. Dong, and B. J. Nelson, "Real-time rigid-body visual tracking in a scanning electron microscope," Int. J. Robot. Res., vol. 28, pp. 498-511, 2009.

[20] C. Ru, Y. Zhang, Y. Sun, Y. Zhong, X. Sun, D. Hoyle, and I. Cotton, "Automated four-point probe measurement of individual nanowires inside a scanning electron microscope," IEEE Trans. Nanotechnol., vol. 10, no. 4, pp. 674-681, Jul. 2011.

[21] D. Jasper and S. Fatikow, "Line scan-based high-speed position tracking inside the SEM," Int. J. Optomechatron., vol. 4, pp. 115-135, 2010.

[22] Y. L. Zhang, M. L. Han, M. Y. Yu, C. Y. Shee, and W. Ang, "Automatic hysteresis modeling of piezoelectric micromanipulator in vision-guided micromanipulation systems," IEEE/ASME Trans. Mechatronics, to be published, DOI: 10.1109/TMECH.2011.2106136.

[23] J. Li, X. Chen, and W. Zhang, "A new approach to modeling system dynamics-in the case of a piezoelectric actuator with a host system," IEEE/ASME Trans. Mechatronics, vol. 15, no. 3, pp. 371-380, Jun. 2010.

[24] H. Liaw and B. Shirinzadeh, "Neural network motion tracking control of piezo-actuated flexure-based mechanisms for micro-/nanomanipulation," IEEE/ASME Trans. Mechatronics, vol. 14, no. 5, pp. 517-527, Oct. 2009.

[25] J. Yi, S. Chang, and Y. Shen, "Disturbance-observer-based hysteresis compensation for piezoelectric actuators," IEEE/ASME Trans. Mechatronics, vol. 14, no. 4, pp. 456-464, Aug. 2009.

[26] S. Bashash and N. Jalili, "Robust adaptive control of coupled parallel piezo-flexural nanopositioning stages," IEEE/ASME Trans. Mechatronics, vol. 14, no. 1, pp. 11-20, Feb. 2009.

[27] X. Liu, J. Tong, and Y. Sun, "A millimeter-sized nanomanipulator with sub-nanometer positioning resolution and large force output," Smart Mater. Struct., vol. 16, pp. 1742-1750, 2007.

[28] K. Kuhnen and H. Janocha, "Inverse feedforward controller for complex hysteretic nonlinearities in smart-material systems," Control Intell. Syst., vol. 29, no. 3, pp. 74-83, 2001.

[29] L. Sun, C. Ru, W. Rong, L. Chen, and M. Kong, "Tracking control of piezoelectric actuator based on a new mathematical model," J.Micromech. Microeng., vol. 14, pp. 1439-1444, 2004

[30] C. Ru and L. Sun, "Improving positioning accuracy of piezoelectric actuators by feedforward hysteresis compensation based on a new mathematical model," Rev. Sci. Instrum., vol. 76, p. 095111, 2005.

[31] W. T. Ang, P. Khosla, and C. Riviere, "Feedforward controller with inverse rate-dependent model for piezoelectric actuators in trajectory-tracking applications," IEEE/ASME Trans. Mechatronics, vol. 12, no. 2, pp. 134142, Apr. 2007.

[32] U.-X. Tan, W. T. Latt, C. Y. Shee, C. Riviere, and W. T. Ang, "Feedforward controller of ill-conditioned hysteresis using singularity-free PrandtlIshlinskii model," IEEE/ASME Trans. Mechatronics, vol. 14, no. 5, pp. 598-605, Oct. 2009.

[33] Q. Xu, Y. Li, and N. Xi, "Design, fabrication, and visual servo control of an XY parallel micromanipulator with piezo-actuation," IEEE Trans. Autom. Sci. Eng., vol. 6, no. 4, pp. 710-719, Oct. 2009.

[34] Y. Li and Q. Xu, "Design and analysis of a totally decoupled flexurebased XY parallel micromanipulator," IEEE Trans. Robot., vol. 25, no. 3, pp. 645-657, Jun. 2009.

[35] Y. Li and Q. Xu, "A totally decoupled piezo-driven XYZ flexure parallel micropositioning stage for micro/nanomanipulation," IEEE Trans. Autom. Sci. Eng., vol. 8, no. 2, pp. 265-279, Apr. 2011.

[36] Y. Li and Q. Xu, "Adaptive sliding mode control with perturbation estimation and PID sliding surface for motion tracking of a piezo-driven micromanipulator," IEEE Trans. Control Syst. Technol., vol. 18, no. 4, pp. 798-810, Jul. 2010.

[37] W. Wang, X. Liu, and Y. Sun, "Contact detection in microrobotic manipulation,” Int. J. Robot. Res., vol. 26, no. 8, pp. 821-828, 2007.

[38] Y. Zhang, B. K. Chen, X. Liu, and Y. Sun, "Autonomous robotic pick-andplace of microobjects," IEEE Trans. Robot., vol. 26, no. 1, pp. 200-207, Feb. 2010.

[39] T. Kasaya, H. T. Miyazaki, S. Saito, K. Koyano, T. Yamaura, and T. Sato, "Image-based autonomous micromanipulation system for arrangement of spheres in a scanning electron microscope," Rev. Sci. Instrum., vol. 75, no. 6, pp. 2033-2042, 2004.

[40] K. Kim, X. Liu, Y. Zhang, and Y. Sun, "Nanonewton force-controlled manipulation of biological cells using a monolithic MEMS microgripper with two-axis force feedback," J. Micromech. Microeng., vol. 18, no. 5, 055013, 2008.

[41] D. Trinh Chu, L. Gih-Keong, J. F. Creemer, and P. M. Sarro, "Electrothermal microgripper with large jaw displacement and integrated force sensors," J. Microelectromech. Syst., vol. 17, no. 6, pp. 1546-1555, 2008.

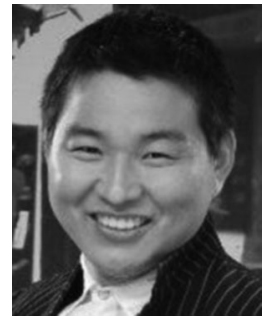

Yan Liang Zhang (M'10) received the B.Eng. degree in computer engineering and the Ph.D. degree in mechanical and aerospace engineering from Nanyang Technological University, Singapore, in 2006 and 2010, respectively.

$\mathrm{He}$ is currently a Postdoctoral Fellow in the Department of Mechanical and Industrial Engineering, University of Toronto, Toronto, ON, Canada. His research interests include cell mechanobiology, robotics automation under optical and electron microand characterization of nanodevices.

scopes for micro/nanomanipulation, and fabrication

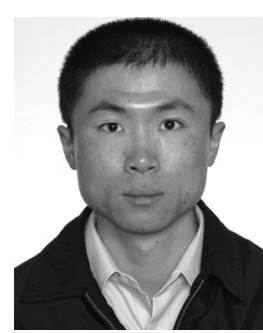

Yong Zhang (S'09) received the B.S. and M.S. degrees in mechatronics engineering from Harbin Institute of Technology, Harbin, China, in 2005 and 2007, respectively. He is currently working toward the Ph.D. degree in the Department of Electrical and Computer Engineering, University of Toronto, Toronto, ON, Canada.

His research interests include the manipulation and characterization of nanomaterials, and the design and fabrication of microelectromechanical systems devices.

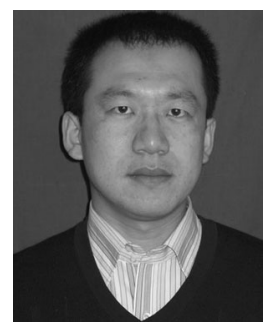

Changhai Ru (M'07) received the Ph.D. degree in mechatronics engineering from Harbin Institute of Technology, Harbin, China, in 2005.

He was a Postdoctoral Fellow in the Department of Mechanical and Industrial Engineering, University of Toronto, Toronto, ON, Canada. He is currently a Professor at the Robotics and Microsystems Center, Soochow University, Jiangsu, China. His research interests include micro/nanomanipulation, nanopositioning technology, and solid-state actuators' driving and control methods.

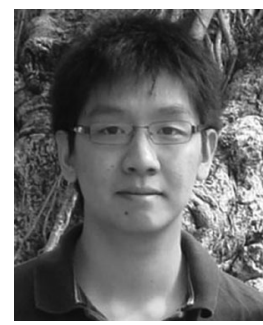

Brandon K. Chen (S'09) received the B.S. degree from Queen's University, Kingston, ON, Canada, in 2007, and the M.S. degree from the University of Toronto, Toronto, ON, Canada, in 2009, both in mechanical engineering. He is currently working toward the Ph.D. degree in the Department of Mechanical and Industrial Engineering, University of Toronto.

His research interests include the design, fabrication, and testing of MEMS-based micro/nanomanipulation tools, and their applications inside SEMs.

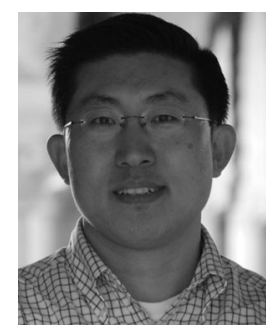

Yu Sun (S'01-M'03-SM'07) received the Ph.D. degree in mechanical engineering from the University of Minnesota, Minneapolis, in 2003.

$\mathrm{He}$ is currently an Associate Professor in the Department of Mechanical and Industrial Engineering and is jointly appointed in the Institute of Biomaterials and Biomedical Engineering and the Department of Electrical and Computer Engineering, University of Toronto, Toronto, ON, Canada. His research interests include micro/nanorobotic manipulation, the design and fabrication of MEMS devices, and the manipulation and characterization of biological cells, biomolecules, and nanomaterials. 